\section{Tivantinib: a new promising mesenchymal-epithelial transition factor inhibitor in the treatment of hepatocellular carcinoma}

\author{
Lorenza Rimassa*, Nicola Personeni', Matteo Simonelli' \& Armando Santoro' \\ 'Medical Oncology \& Hematology Unit, Humanitas Cancer Center, Istituto Clinico Humanitas, IRCCS \\ Via Manzoni 56, 20089 Rozzano (Milano), Italy \\ *Author for correspondence: Tel.: +39 0282244573 - Fax: +39 0282244590 m Iorenza.rimassa@ \\ cancercenter.humanitas. it
}

Tivantinib (ARQ 197) is an orally administered, selective small molecule that inhibits mesenchymal-epithelial transition factor (MET) via a novel, ATP-independent binding mechanism. Preclinical studies demonstrated that tivantinib has a broad-spectrum anti-tumor activity, especially in cells expressing high levels of MET. A randomized Phase II study in second-line hepatocellular carcinoma showed statistically significant improvement in time to progression with tivantinib compared to a placebo. Noteworthy, a significant pronounced benefit in time to progression and overall survival was observed in MET-high patients. In addition, MET expression was defined as a negative prognostic factor. The most frequent adverse events were hematologic events. A Phase III study in the MET-high hepatocellular carcinoma is actively recruiting patients. Phase II and III studies in non-small-cell lung cancer and colorectal cancer are ongoing.

Hepatocellular carcinoma (HCC) is the fifth most common malignancy worldwide, representing the third leading cause of cancer-related deaths, with a rising incidence, particularly in Western countries [1]. Therapeutic approaches including hepatic resection, liver transplantation and locoregional therapies play a major role in the clinical management of HCC [2-4]. However, the high frequency of tumor recurrence and patients diagnosed at advanced stage requires systemic drug intervention. Current standard of care for patients with advanced HCC and a preserved liver function (Child-Pugh class A) is treatment with sorafenib, a small-molecule multitargeted receptor tyrosine kinase (TK) inhibitor (TKI), which blocks several receptor TKs involved in tumor cell proliferation and angiogenesis $[5,6]$. Other emerging targets for the treatment of advanced HCC include the mesenchymal-epithelial transition factor (MET) receptor TK and its sole ligand, HGF, which represent one of the most frequently deregulated signaling pathways in human cancer $[7,8]$. Stimulation of the HGF/MET signaling axis leads to the activation of multiple intracellular effectors, such as the Src/FAK, Ras/Raf/MEK/ERK and PI3K/Akt, which promote tumor cell growth, survival, migration and invasion, as well as tumor angiogenesis and metastasis. The aberrant activation of $M E T$ can occur through multiple mechanisms, including gene amplification, transcriptional upregulation, point mutations, and HGF-mediated autocrine or paracrine activation $[7,8]$. Overexpression or enhanced activation of HGF or MET has been reported in a variety of human cancer types and correlates with increased metastases, cancer aggressiveness and poor prognosis [9]. Several studies demonstrated that the deregulation of the HGF/MET network is a common feature in HCC development and progression. MET overexpression is observed in $20-48 \%$ of human HCC samples and correlates with a significantly shorter 5 -year survival rate [10-12]. A predictive model established according to $M E T$-dependent gene expression signatures was able to define a subset of HCC characterized by an aggressive phenotype and poor prognosis [13]. In addition, the knockdown of MET by antisense RNA or RNAi strategies abrogated the in vitro and in vivo growth of HCC cells $[14,15]$. In this review, we discuss the preclinical and clinical data of tivantinib, an oral selective MET inhibitor, focusing the attention on HCC studies.

\section{Overview of the market}

Sorafenib was approved in 2008 for first-line systemic treatment of HCC, based on results of the SHARP trial, which reported a significant increase in survival and time to radiological progression over placebo [5]. The most frequent adverse events (AEs) were diarrhea,

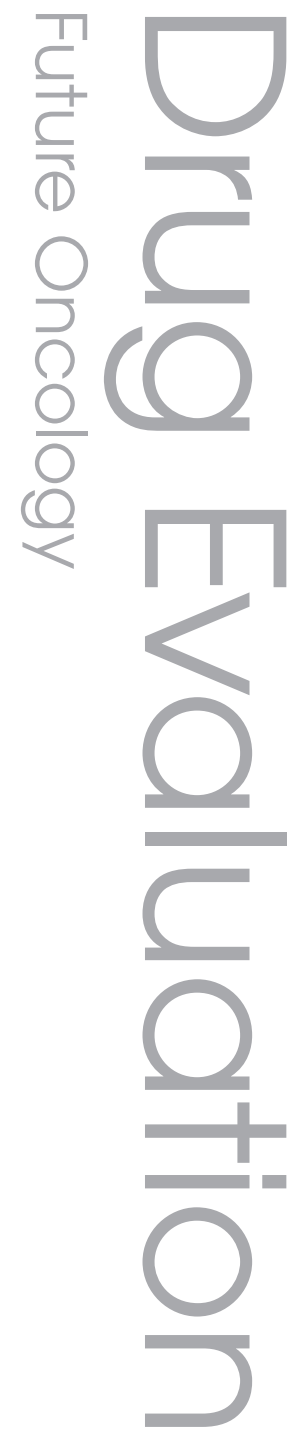

\section{Keywords}

- hepatocellular carcinoma

- immunohistochemistry

- locally advanced/

metastatic disease $=$ MET expression $\approx$ MET inhibitor - second line = tivantinib

\section{Future :\%: Medicine part of}


hand-foot skin reaction, fatigue and weight loss, all of which were often manageable [5]. Although safety was not a major issue in the development of sorafenib, this might not be the case for other compounds where toxicities related to concomitant cirrhosis represent a concrete bottleneck, hampering their clinical development. In fact, despite promising data from two independent Phase II trials [16,17], a Phase III trial comparing sunitinb to sorafenib in patients with advanced HCC was discontinued due to concerns regarding safety profile, in addition to a lack of efficacy [18]. Furthermore, efficacy may also depend on the patient's characteristics and geographical origins. For instance, a parallel trial was concomitantly conducted to the SHARP trial [5] in the Asia-Pacific region [6], where HBV-related disease and more advanced stages are more prevalent compared with western countries. Results of this trial confirmed the magnitude of the benefit of sorafenib compared with placebo (hazard ratio [HR]: 0.68) [6].

In patients who are resistant, refractory or intolerant to sorafenib, no effective therapeutic options currently exist outside of clinical trials, and several targeted drugs are under evaluation in Phase II and III studies for second-line treatment. Most of these agents are designed to inhibit angiogenesis, an important process in HCC pathogenesis and progression, and include monoclonal antibodies (moAbs) or intracellular TKI. Brivanib, which targets both VEGF receptor (VEGFR) and FGF receptor, had the theoretical potential to overcome resistance to sorafenib. Nevertheless, a Phase III trial of brivanib versus placebo reported a median overall survival (OS) for the brivanib arm of 9.4 versus 8.2 months for the placebo arm (HR: 0.89; $\mathrm{p}=0.33$ ), thereby not meeting its primary end point [19]. Two other drugs are currently being tested against placebo in Phase III trials for regulatory approval. These include ramucirumab, a moAb against the VEGFR [101], and everolimus, an mTOR blocker approved for kidney cancer therapy [102]. Classifications of cancer subtypes based on molecular biomarkers have allowed for the identification of distinct oncogenic drivers, which may become both therapeutic targets and potential predictors of response. Three HCC subtypes were characterized by gene expression profiling [20], however, no molecular biomarker has been derived to predict response to targeted therapies and, specifically, to sorafenib. In a Phase II trial of sorafenib [21], the pretreatment levels of phosphorylated ERK were significantly correlated with time to progression
(TTP). Despite preclinical evidences of a correlation between phosphorylated ERK levels and sensitivity to sorafenib [22], these findings could not be further validated. Recently, in a correlative study of the SHARP trial [5], ten candidate plasma biomarkers were analyzed in both the placebo and the sorafenib arm. The angiogenesis biomarkers, Ang2 and VEGF, were reported to be independent prognostic markers of survival. Nevertheless, when authors tested the interaction between sorafenib treatment and each biomarker level at baseline, no biomarker was found to predict the benefit deriving from sorafenib over placebo $[23,24]$.

The limited understanding of potential oncogenic drivers in HCC, partially related to the high molecular heterogeneity of the disease, has hampered the implementation of therapeutic strategies already pursued in other solid tumors. On the other hand, clinical experience with sorafenib indicates that simultaneous blockade of a wide kinase spectrum might represent an alternative approach to tackle, from a therapeutic standpoint, the heterogeneous molecular landscape of HCC.

\section{Tivantinib}

Tivantinib (formerly known as ARQ 197) is a highly selective, orally administered, non-ATP competitive inhibitor of the MET receptor TK, with an inhibitory constant $\left(\mathrm{K}_{\mathrm{i}}\right)$ of $355 \mathrm{nmol} / \mathrm{l}$ in biochemical assays (ArQule, Inc. [103]; Daiichi Sankyo, Inc. [104]; Kiowa Hakko Kirin, Co., Ltd). In a large kinase panel screen, tivantinib has been shown to be 10-100-times more selective for MET than 229 other kinases tested. This agent presents a novel binding mechanism of action that facilitates the stabilization of an inactive nonphosphorylated configuration of MET, inhibiting autoactivation of the kinase.

\section{Chemistry}

Tivantinib (chemical formula $(3 \mathrm{R}, 4 \mathrm{R})$ 3-(5,6-dihydro-4H-pyrrolo[3,2,1-ij] quinolin1-yl)-4-(1H-indol-3-yl)pyrrolidine-2,5-dione) (FIgUre 1) is the most advanced representative of a newly discovered class of trans 3,4-disubstituted pyrrolidine-2,5-diones, which acts as a non-ATP competitive inhibitor of both constitutive and ligand-mediated MET phosphorylation [25].

\section{Pharmacodynamics \& preclinical studies Cancer cell line/in vitro studies}

Tivantinib was discovered by a phenotypedriven process, using pathway-based assays in metastatic cancer cell lines. To assess the effect 
of tivantinib on MET phosphorylation and its downstream effectors (Akt, ERK1/2 and STAT3), a wide range of human cancer cell lines (i.e., HT29, MKN-45, MDA-MB-231, and NCI-H441) were used [25]. Following a 72-h in vitro exposure to tivantinib, both constitutive and HGF-mediated MET activation were inhibited with an $\mathrm{IC}_{50}$ of $100-300 \mathrm{nmol} / \mathrm{l}$. Moreover, the pharmacodynamic effects of tivantinib on cancer cells were fully comparable to those obtained through siRNA-mediated MET depletion. In a standard cytotoxicity assay, cancer cell lines expressing activated MET (i.e., MKN-45 and HT29) were strongly sensitive to tivantinib and showed caspase-dependent apoptosis, whereas cancer cells without detectable MET proteins (SK-MEL-28, NCI-H661, NCI-H446) were markedly less sensitive (ten-to-100-fold) [25]. These findings confirm the hypothesis that tivantinib exerts its antiproliferative and proapoptotic effects directly through the inhibition of MET and MET-dependent signaling pathway. In vitro assays revealed that the combination of tivantinib and sorafenib had synergistic or additive cytotoxicity against a large panel of human cancer cell lines (five of seven HCC lines tested) [26]; this effect was also noted for the combination of tivantinib and gemcitabine, especially with an intermittent schedule [27].

\section{In vivo studies}

To test the anti-tumor activity of tivantinib in vivo, three xenograft mouse models of human colon, pancreas and breast cancers (HT29, MKN-45, MDA-MB231) were evaluated. Following an oral administration of tivantinib at $200 \mathrm{mg} / \mathrm{kg}$ (serum concentrations higher than $300 \mathrm{nM}$ for up to $12 \mathrm{~h}$ ), significant tumor growth reductions of $45-79 \%(\mathrm{p}<0.05)$ were observed in all these xenograft models without significant body weight changes [25]. Pharmacodynamically, a single oral dose of $200 \mathrm{mg} / \mathrm{kg}$ of tivantinib induced a dramatic reduction in immunodetectable levels of phosphorylated MET in human colon xenograft tumors (HT29) $24 \mathrm{~h}$ after dosing. Consistent with in vitro data, tumor xenografts were exposed to sustained plasma levels of tivantinib $(1.3 \mu \mathrm{mol} / \mathrm{l} 10 \mathrm{~h}$ after dosing, value greater than threefold the inhibitory constant of tivantinib for MET) [25]. A dosedependent antimetastatic activity of tivantinib was demonstrated in a humanized mouse model of metastatic breast cancer. At a daily dose of $120 \mathrm{mg} / \mathrm{kg}$, tivantinib prevented the onset and progression of bone metastases and reduced the tumor-induced osteolysis [28]. Furthermore,

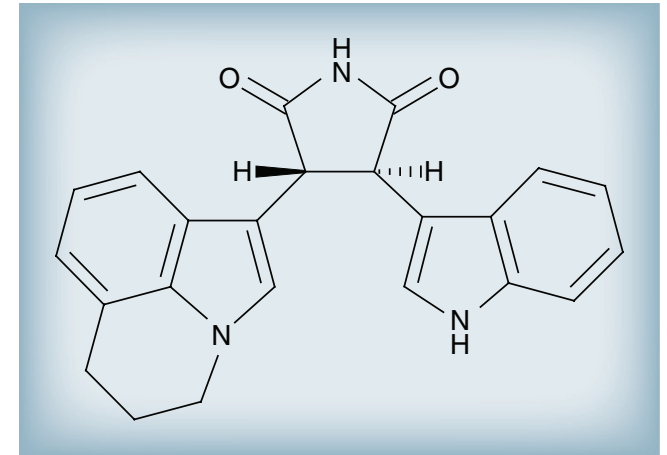

Figure 1. Tivantinib. Tivantinib is the most advanced representative of a newly discovered class of trans-3,4-disubstituted pyrrolidine2,5-diones, which acts as a non-ATPcompetitive inhibitor of both constitutive and ligand-mediated mesenchymal-epithelial transition factor phosphorylation.

the combination of tivantinib and sorafenib demonstrated synergistic activity in a xenograft model of non-small-cell lung cancer (NSCLC; NCI-H522), supporting the rationale for clinical development of this combination [26].

\section{Preclinical pharmacokinetics}

Tivantinib is rapidly metabolized by CYP2C19 (half-life $=2.8 \mathrm{~min}$ ) and moderately metabolized by CYP3A4 (half-life $=16.3 \mathrm{~min}$ ), without inhibiting any of the major CYP450 enzymes tested [29]. As shown by metabolic studies in animals and human hepatocytes, the oxidative biotransformation represents the primary metabolic pathway of tivantinib with an oral bioavailability $\geq 20 \%$ [29].

\section{Clinical pharmacokinetics}

Clinical pharmacokinetic (PK) parameters of tivantinib were evaluated in three Phase I studies as monotherapy and in three combination studies with erlotinib, sorafenib and gemcitabine [27,30-34]. Tivantinib was administered at doses ranging from 10 to $400 \mathrm{mg}$ twice daily (b.i.d.) and in three different active pharmaceutical ingredient forms (amorphous, crystalline $\mathrm{A}$, and crystalline B). Crystalline B resulted in being the most stable and is currently being used in patients at $360 \mathrm{mg}$ b.i.d. Overall PK profile of tivantinib was characterized by a moderate rate of absorption (peak 2-4 h after dosing), followed by a linear decrease in plasma concentrations approximately $4 \mathrm{~h}$ after dosing. Despite a wide interpatient variability, systemic tivantinib exposure generally increased over the dosing period. However, in most of cases, plasma levels of tivantinib grew less than proportionally with increasing dose. No relationships between drug-related 
AEs, dose, baseline demographic variables and extent of tivantinib exposure were reported. CYP2C19*2 and CYP2C19*3 single nucleotide polymorphisms, relatively common in Asian subjects (35\% allele frequency), resulted in enzyme deficiency and poor metabolism of tivantinib [31]. In combination with other agents, PK parameters of tivantinib appeared similar to those reported in monotherapy studies, indicating the absence of drug interactions [27,33,34].

\section{Phase I trials}

The initial safety and tolerability of tivantinib as a single agent has been explored in two Phase I, dose-escalation trials in adult patients with advanced solid tumors (Table $\mathbf{1}$ ).

In the first clinical evaluation in humans, two different dosing regimens of tivantinib were studied. After a favorable safety profile of an intermittent schedule (2 weeks on/1 week off) had been fully established, a continuous dosing regimen was introduced in a further 42 patients. Drug-related AEs of any grade were reported in 37 (46.8\%) of the 79 patients enrolled [30]. Only two patients experienced dose-limiting toxicities (DLTs), including leukopenia, neutropenia, thrombocytopenia, vomiting and dehydration. Both of these patients received continuous dosing of tivantinib at $360 \mathrm{mg}$ b.i.d. and the maximum tolerated dose was not reached because less than $33 \%$ of patients experienced DLTs at any given dose. The most common $(\geq 5 \%)$ drug-related AEs included fatigue (13.9\%), nausea $(13.9 \%)$, vomiting $(10.1 \%)$, anemia $(7.6 \%)$ and diarrhea $(6.3 \%)$. The fatigue and gastrointestinal disorders were generally mild to moderate in severity and unrelated to dose, while an apparent dose-related increase in the frequency of anemia was reported, particularly at the $360 \mathrm{mg}$ b.i.d. dose level. Among the 62 patients assessable for clinical activity, three patients $(4.8 \%)$ achieved a partial response (PR), 38 patients $(62 \%)$ showed stable disease (SD), whereas 20 patients $(33 \%)$ progressed. Sixteen patients $(25 \%)$ received tivantinib for more than 24 weeks and the median progression-free survival (PFS) was 12.1 weeks [30].

In order to identify the recommended Phase II dose (RP2D) using a continuous b.i.d. dosing schedule, a second Phase I study was conducted [30]. A total of 51 patients received tivantinib at doses ranging from 100 to $400 \mathrm{mg}$ b.i.d. DLTs included grade 3 fatigue (200 mg b.i.d.; $\mathrm{n}=1$ ), grade 3 mucositis, palmar-plantar erythrodysesthesia, hypokalemia (400 mg b.i.d.; $\mathrm{n}=1$ ) and grade 3 febrile neutropenia (400 mg b.i.d.; $\mathrm{n}=2$ ). All DLTs were resolved within 2 weeks of drug discontinuation. The maximum tolerated dose/RP2D of tivantinib was initially established as $300 \mathrm{mg}$ b.i.d. (amorphous formulation), but was subsequently adjusted to $360 \mathrm{mg}$ b.i.d. (PK equivalent) with the crystalline formulation [31]. Tivantinib was well tolerated with the most common toxicities being grade $1 / 2$ fatigue (15.7\%), nausea $(13.7 \%)$ and vomiting (11.8\%). Stable disease $\geq 4$ months was achieved in 14 patients (27\%), with minor tumor responses observed in gastric and Merkel cell carcinomas. Consistent with in vitro studies, pharmacodynamic analyses demonstrated that tivantinib administration decreased intratumoral phosphorylated and total MET levels, as well as phosphorylated FAK, with the concomitant induction of apoptosis as shown by terminal deoxynucleotidyl transferase dUTP nick end-labeling assays. Circulating endothelial cells declined significantly after treatment with tivantinib, while no significant changes in dynamic contrast-enhanced MRI parameters were observed [31].

In view of the growing evidence regarding the critical role of an aberrant HGF/MET network in HCC development and progression, a Phase Ib study was undertaken to evaluate the safety of tivantinib in 21 refractory patients with HCC and concomitant cirrhosis [32]. No drug-related worsening of liver function was observed and, therefore, tivantinib $360 \mathrm{mg}$ b.i.d. was deemed the RP2D. Neutropenia was the most common drug-related AE and represented the primary reason for dose reduction, interruption or discontinuation. The frequency of any grade $(52 \%)$ or severe (grade $\geq 3 ; 38 \%$ ) neutropenia was markedly higher when compared with other dose-escalation trials, requiring a careful follow-up of hematologic toxicities in this population. Preliminary evidence of clinical activity in terms of SD was observed in nine of the 16 evaluable patients with a median duration of 4.6 months [32].

On the basis of strong preclinical evidences, dual EGFR-MET inhibition has been proposed as a strategy to overcome primary and secondary resistance to EGFR inhibitors in the clinical setting [35]. A total of 32 patients with metastatic solid tumors were treated with an escalating doses of tivantinib (120, 240 and $360 \mathrm{mg}$ b.i.d.) in combination with a fixed dose of erlotinib (150 mg daily) [33]. Single or multiple intrasubject dose escalations were allowed in the absence of DLT through one cycle of therapy. The combination was well tolerated, with rash, nausea, abdominal pain, diarrhea, 


\section{Table 1. Phase I/II clinical trials of tivantinib.}

\begin{tabular}{|c|c|}
\hline Study (year) & Trial details \\
\hline \multicolumn{2}{|c|}{ Phase I studies as a single agent } \\
\hline $\begin{array}{l}\text { Rosen et al. } \\
\text { (2011) }\end{array}$ & $\begin{array}{l}\text { Phase I dose-escalation study in } 79 \text { patients with } \\
\text { advanced solid tumors. Two different dose schedules } \\
\text { (intermittent and continuous) and } 13 \text { dose levels, } \\
\text { ranging from } 10 \text { to } 360 \text { mg b.i.d. Three different } \\
\text { active pharmaceutical ingredient forms (amorphous, } \\
\text { crystalline A and crystalline B) }\end{array}$ \\
\hline
\end{tabular}

Yap et al. Phase I dose-escalation study in 51 patients with (2011) advance solid tumors; tivantinib doses ranging from 100 to $400 \mathrm{mg}$ b.i.d.

Santoro et al. Phase lb study in 21 patients with HCC and (2013) concomitant CP class A $(n=17)$ or $B(n=4)$ liver cirrhosis

\section{Phase I studies in combination with other agents}

Goldman et al. Phase I dose-escalation study of tivantinib (120, 240 (2012) and $360 \mathrm{mg}$ b.i.d.) in combination with a fixed dose of erlotinib (150 mg daily) in patients with advanced solid tumors

Adjei et al. Phase I dose-escalation study of tivantinib (240 and (2010) $360 \mathrm{mg}$ b.i.d.) in combination with sorafenib (200 and $400 \mathrm{mg}$ b.i.d.)

Martel et al. Expansion phase in $20 \mathrm{HCC}$ patients: 10 patients (2012) treated at the RP2D and 10 with tivantinib at $240 \mathrm{mg}$ b.i.d.

Pant et al. Phase lb dose-escalation study of tivantinib (120, 240 (2010) and $360 \mathrm{mg}$ b.i.d.) in combination with gemcitabine. Different dosing schedules: continuous, 3 weeks on/1 week off, 2 weeks on/1 week off, and 5 out of 7 days weekly

\section{Outcome}

Ref.

MTD not reached

[30]

Most common drug-related AEs included fatigue

$(13.9 \%)$, nausea $(13.9 \%)$, vomiting $(10.1 \%)$, anemia

(7.6\%) and diarrhea (6.3\%)

Tivantinib $360 \mathrm{mg}$ b.i.d. well tolerated, with mild-to-moderate toxicities

Tivantinib MTD/RP2D established at 360 mg b.i.d. with the crystalline formulation

No drug-related worsening of liver function

Tivantinib 360 mg b.i.d. deemed the RP2D. Higher frequency of neutropenia

No formal MTD established. Tivantinib 360 mg b.i.d. in combination with erlotinib $150 \mathrm{mg}$ considered as the RP2D. DCR $46.8 \%$; $6 / 8$ patients with NSCLC with long-lasting SD

No formal MTD established. Tivantinib 360 mg b.i.d. in combination with sorafenib $400 \mathrm{mg}$ b.i.d. considered as the RP2D. Preliminary evidence of anticancer activity in patients with RCC, HCC or melanoma

ORR 10\% (1 CR, 1 PR), DCR 75\%. TTP for CP class A patients $(n=14) 7.3$ months

RP2D identified as tivantinib $360 \mathrm{mg}$ b.i.d. continuously with gemcitabine $1000 \mathrm{mg} / \mathrm{m}^{2}$ weekly for 3 weeks on/1 week off. Following in vitro experiments, patients treated with a fixed gemcitabine schedule and pulsatile tivantinib. Promising anti-tumor activity in patients with breast, ovarian, uterine carcinoma and thoracic tumors

\section{Phase II studies}

Santoro et al.

107 patients with locally advanced or metastatic

Increased TTP for the ITT population (6.9 vs

(2012) HCC, failing first-line systemic therapy, randomized to tivantinib (71 patients) or placebo (36 patients);

6.0 weeks). Most pronounced clinical benefit for MET-high patients, in terms of TTP (11.7 vs crossover allowed at radiographic disease progression 6.1 weeks), PFS and OS (7.2 vs 3.8 months) (23 patients)

Sequist et al. 167 previously treated patients with NSCLC

(2011) randomized to erlotinib with or without tivantinib

Eng et al. (2012) 122 patients with KRAS wild-type pretreated mCRC treated with tivantinib plus irinotecan and cetuximab
Prolonged adjusted PFS (adjusted HR: 0.61) for 117 patients with nonsquamous histology

In the Phase I trial portion ORR 44\%; among three patients with MET-high tumors, 2 PR and 1 SD. Results of the Phase II trial are awaited

AE: Adverse event; b.i.d.: Twice daily; CP: Child-Pugh; CR: Complete response; DCR: Disease control rate; HCC: Hepatocellular carcinoma; HR: Hazard ratio; ITT: Intent-to-treat; mCRC: Metastatic colorectal cancer; MET: Mesenchymal-epithelial transition factor; MTD: Maximum tolerated dose; NSCLC: Non-small-cell lung cancer; ORR: Overall response rate; OS: Overall survival; PFS: Progression-free survival; PR: Partial response; RCC: Renal cell carcinoma; RP2D: Recommended Phase II dose; SD: Stable disease; TTP: Time to progression.

bradycardia and anemia being the most common drug-related AEs, mostly mild to moderate in severity. Two DLTs occurred at the $360 \mathrm{mg}$ b.i.d. dose level. One patient developed grade 4 neutropenia on day 15 , while a patient developed grade 4 neutropenia and leukopenia plus grade 3 thrombocytopenia on day 14 . Two patients experienced arrhythmic serious AEs including sinus bradycardia and sick sinus syndrome. In the absence of a formally established maximum tolerated dose, given the favorable safety profile and preliminary signs of clinical activity, tivantinib 
$360 \mathrm{mg}$ b.i.d. in combination with erlotinib $150 \mathrm{mg}$ daily was considered the RP2D. One PR (3.1\%) was seen in a microcystic adnexal carcinoma and SD occurred in 14 patients (43.8\%). Six of the eight subjects with NSCLC achieved SD with a duration of 3-33 months. Of note, five out of six patients who had previously progressed to erlotinib monotherapy achieved SD with the combination therapy [33]. These encouraging results prompted further evaluation of this combination in NSCLC patients.

As reported above, the combination of tivantinib and sorafenib revealed synergistic and additive antiproliferative activity in preclinical studies [26]. To verify the safety and tolerability of this combination in the clinical setting, a Phase I dose-escalation study was performed in patients with advanced solid tumors [34]. Following the determination of the RP2D, an expansion cohort phase for patients with renal cell carcinoma, HCC, breast cancer, NSCLC and melanoma was opened. In the absence of DLTs, dosing was increased to the full single agent doses of both drugs (tivantinib $360 \mathrm{mg}$ b.i.d. + sorafenib $400 \mathrm{mg}$ b.i.d.) considered as the RP2D. A total of 62 patients were enrolled. The most common drug-related AEs of any grade were diarrhea $(40 \%)$, rash $(40 \%)$, fatigue (39\%), anorexia (31\%), hypophosphatemia (21\%) and stomatitis (21\%). A total of six serious AEs (all grade 3 ) were reported in four patients, including angina unstable, atrial fibrillation, diarrhea, febrile neutropenia, neutropenia and pneumonia. Preliminary evidence of anticancer activity was observed in patients with renal cell carcinoma, HCC and melanoma [36-38].

Among the 20 patients with HCC of the expansion phase, ten were treated at the RP2D and ten with tivantinib dose reduced at $240 \mathrm{mg}$ b.i.d. after safety review of the HCC single-agent Phase II study. In this subgroup of patients, one complete response (CR), one PR and 12 SD were observed, accounting for an overall response rate (ORR) of $10 \%$ and a disease control rate of 75\%. Interestingly, responses (one CR plus one $\mathrm{PR}$ ) and stabilizations (three SD) were reported in eight HCC patients previously treated with sorafenib, suggesting that the combined inhibition of MET and angiogenic signals may have therapeutic potential in this setting. Of note, the patient who experienced CR presented a high MET expression [36].

The combination of tivantinib and gemcitabine was tested in a multicenter Phase Ib trial. A total of 65 patients with advanced solid tumors received tivantinib at escalating doses $(120 \mathrm{mg}$ b.i.d., $240 \mathrm{mg}$ b.i.d. and $360 \mathrm{mg}$ b.i.d.) with different dosing schedules (continuous, 3 weeks on/ 1 week off, 2 weeks on/1 week off, and 5 of 7 days weekly) [27]. During the dose-escalation, drug-related AEs were reported in 57 patients (87.7\%) with thrombocytopenia, anemia, neutropenia, fatigue and nausea being the most common toxicities. No DLTs were observed and the RP2D was identified as tivantinib $360 \mathrm{mg}$ b.i.d. continuously with gemcitabine $1000 \mathrm{mg} / \mathrm{m}^{2}$ weekly for 3 weeks on $/ 1$ week off. After encouraging clinical responses with the interrupted schedule, in vitro experiments demonstrated an advantage in growth inhibition for alternating or sequential schedules. In view of these results, the protocol was amended to treat patients with a fixed gemcitabine schedule (days 8, 15 and 22) and intermittent tivantinib (days 2-6, 9-13, 16-20 and 23-27) [27]. Promising anti-tumor activity was observed in patients with breast, ovarian, uterine carcinoma and thoracic tumors, including patients who progressed after prior gemcitabine therapy $[39,40]$. Expanded cohorts in these gemcitabine-sensitive tumor types are currently enrolling patients.

\section{Clinical efficacy}

Phase II study in HCC

The results of a multicentric, international, randomized, double-blind, placebo-controlled Phase II study of tivantinib in second-line unresectable HCC were recently published (Table 1) [41]. A total of 107 patients with cito-histologically confirmed locally advanced or metastatic HCC, progression or intolerance to first-line systemic therapy (103 sorafenib, four sunitinib), ChildPugh class A cirrhosis, Eastern Cooperative Oncology Group performance status $0-1$ and no prior liver transplant (stratified by performance status and presence/absence of vascular invasion) were randomized $2: 1$ to tivantinib or placebo, 71 patients on tivantinib and 36 on placebo. At radiographic progression, 23 patients on placebo crossed over to open-label tivantinib.

The primary end point was TTP in the intentto-treat population, assessed by a central independent radiologist according to the Response Evaluation Criteria in Solid Tumors (version 1.1) [42]. Tumor measurements were performed every 6 weeks. Secondary end points included PFS, OS, ORR, disease control rate, crossover ORR, safety and pharmacokinetics. Furthermore, subgroup analyses were preplanned to evaluate efficacy according to MET status, viral status (HBV and/or HCV) and duration of prior systemic therapy. 
Patients were enrolled from October 2009 to August 2011 in Europe and North America. After 57 patients were enrolled (38 in the tivantinib arm), a higher-than-expected incidence of grade $>3$ neutropenia was observed. As a consequence, the tivantinib dose of $360 \mathrm{mg}$ b.i.d. was reduced to $240 \mathrm{mg}$ b.i.d. in all patients and a modified dose reduction schema was implemented. Patients' characteristics were well-balanced among the study arms.

The study met its primary end point of TTP for the intent-to-treat population. Treatment with tivantinib produced a statistically significant $56 \%$ improvement in TTP, with a median of 6.9 weeks compared with 6.0 weeks (HR: 0.64; 90\% CI: 0.43-0.94; $\mathrm{p}=0.04)$. PFS was consistent with TTP with a HR of 0.67 (95\% CI: 0.44-1.04) and a p-value of 0.06. Results in OS were not statistically significant. Of note, efficacy was comparable at 240 and $360 \mathrm{mg}$ b.i.d., and at $240 \mathrm{mg}$ b.i.d. fewer patients came off therapy or died due to side effects.

MET expression was evaluated by immunohistochemistry (IHC) assay using the anti-total MET (SP44) rabbit monoclonal antibody developed by Ventana Medical Systems (AZ, USA) at an independent central laboratory, after randomization and prior to unblinding. MET-high was defined as $>50 \%$ of tumor cells with moderate or strong $(2+$ or $3+)$ staining intensity. A total of 30 patients ( 22 in the tivantinib and eight in the placebo arms) were not evaluable for MET expression due to tissue sample inadequacy, $45 \%$ of patients on tivantinib and $54 \%$ of patients on placebo were classified as MET-high. No significant imbalances were found in patients' characteristics when grouped by MET status.

On placebo, median OS was 3.8 months in MET-high patients compared with 9.0 months in MET-low patients (HR: 2.94; 95\% CI: $1.16-7.43 ; \mathrm{p}=0.02)$, demonstrating the prognostic effect of MET.

The most pronounced clinical benefits in this study were observed in MET-high patients, the analysis of which was prespecified in the statistical analysis plan. These patients experienced a $133 \%$ improvement in TTP, with a median of 11.7 weeks compared with 6.1 weeks (HR: 0.43; 95\% CI: 0.19-0.97; $\mathrm{p}=0.03)$. PFS was consistent with TTP analysis, with a HR of 0.45 (95\% CI: $0.21-0.95)$ and a p-value of 0.02 . Disease control rate was $50 \%$ (range from 28 to $72 \%$ ) on tivantinib versus $20 \%$ (range from 4 to $48 \%$ ) on placebo. Median OS was 7.2 months on tivantinib versus 3.8 months on placebo (HR: 0.38 ; 95\% CI: 0.18-0.81; $\mathrm{p}=0.01$ ), representing a $163 \%$ improvement in OS. Of note, eight of the 15 placebo patients crossed over to open-label tivantinib and five remained on therapy for at least 6 weeks. In the MET-low population, efficacy results were statistically similar between the tivantinib and placebo arms.

Based on these results, an international Phase III study in second-line MET-high HCC is actively recruiting patients, to provide a new therapeutic option for this prevalent worldwide cancer.

\section{Safety \& tolerability}

In the Phase II HCC study, the most commonly reported AEs (all grade) were asthenia, neutropenia, decreased appetite and anemia (Table 2). Among patients treated with tivantinib, the most frequent grade 3-5 AEs were neutropenia, observed in $21 \%$ of the patients at the dose of $360 \mathrm{mg}$ b.i.d. but in only $6 \%$ of the patients at the dose of $240 \mathrm{mg}$ b.i.d., and anemia, observed in $16 \%$ of the patients at the dose of $360 \mathrm{mg}$ b.i.d. and $9 \%$ of the patients at the dose of $240 \mathrm{mg}$ b.i.d. Five patients (four at the dose of $360 \mathrm{mg}$ b.i.d.) with neutropenia also developed sepsis. Less frequent AEs included thrombocytopenia and hepatic failures, the latter of which were considered non-drug-related. Overall, AEs were similar in tivantinib and placebo arms, except for a higher incidence of hematologic events in the tivantinib arm and a manageable safety profile was observed with the dose of $240 \mathrm{mg}$ b.i.d.

\section{Other clinical indications}

MET receptor activation is associated with poor prognosis and EGFR TKI resistance in NSCLC $[43,35]$. In a Phase II international randomized trial, 167 previously treated patients with EGFR TKI-naive NSCLC were randomized to receive erlotinib (150 mg daily) with or without tivantinib (360 mg b.i.d.) (Table 1) [44]. PFS analysis, the primary end point of the study, favored the tivantinib arm, particularly in the preplanned adjusted analysis on the 117 patients with nonsquamous histology (HR: 0.61; 95\% CI: 0.39-0.98; $\mathrm{p}=0.04)$. A cohort of 15 patients with KRAS mutations achieved an even more significant benefit in PFS (HR: 0.18; 95\% CI: 0.05-0.70; $\mathrm{p}<0.01$; interaction $\mathrm{p}=0.006)$. Interestingly, patients on the tivantinib arm had a longer time-to-new metastatic lesions (HR: 0.49; 95\% CI: 0.31-0.78; p = 0.01). Importantly, AEs were similar in both study arms and no unexpected toxicity was observed. 
Table 2. Adverse events in the second-line randomized Phase II hepatocellular carcinoma study.

Preferred term

Tivantinib $240 \mathrm{mg}$ b.i.d. $(n=33)$

\section{Patients (\%)}

Tivantinib 360 mg b.i.d. ( $n=38)$
Placebo

( $n=36)$

\begin{tabular}{|c|c|c|c|c|c|c|}
\hline & & & & \\
\hline & All grades & Grade 3-5 & All grades & Grade 3-5 & All grades & Grade 3-5 \\
\hline All AEs & 100 & 49 & 95 & 55 & 86 & 44 \\
\hline \multicolumn{7}{|c|}{ Most common (>10\%) AEs } \\
\hline Asthenia & 42 & 6 & 13 & 3 & 19 & 3 \\
\hline Neutropenia $^{+}$ & 21 & 6 & 29 & 21 & 6 & 0 \\
\hline Decreased appetite & 27 & 0 & 24 & 3 & 17 & 6 \\
\hline Anemia & 21 & 9 & 21 & 16 & 6 & 0 \\
\hline Fatigue & 12 & 3 & 29 & 8 & 28 & 6 \\
\hline Peripheral edema & 24 & 6 & 18 & 0 & 17 & 0 \\
\hline Ascites & 30 & 6 & 8 & 0 & 14 & 0 \\
\hline Diarrhea & 9 & 0 & 24 & 0 & 25 & 3 \\
\hline Abdominal pain & 9 & 3 & 16 & 0 & 19 & 3 \\
\hline Cough & 12 & 0 & 13 & 0 & 14 & 3 \\
\hline Pyrexia & 9 & 0 & 13 & 0 & 14 & 0 \\
\hline \multicolumn{7}{|c|}{ Other AEs of particular relevance } \\
\hline Thrombocytopenia & 9 & 6 & 11 & 5 & 0 & 0 \\
\hline Hepatic failure & 6 & 6 & 5 & 5 & 0 & 0 \\
\hline \multicolumn{7}{|l|}{ SAES } \\
\hline AEs resulting in death & 21 & & 16 & & 28 & \\
\hline All SAEs (all grades) & 39 & & 29 & & 39 & \\
\hline
\end{tabular}

Consistent with the literature, at a retrospective, central IHC analysis in archival tissue samples, MET expression rate was found to be higher in nonsquamous $(76 \%)$ than in squamous $(12 \%)$ NSCLC $[45,46]$. The 25 nonsquamous, MET-high patients on tivantinib plus erlotinib showed an improvement in both PFS (HR: 0.58; $\mathrm{p}=0.28)$ and $\mathrm{OS}(\mathrm{HR}: 0.46 ; \mathrm{p}=0.21$ ) compared with patients on erlotinib alone, while no detrimental effects were observed in patients with MET-low, nonsquamous tumors.

Results are expected from two randomized Phase III trials on nonsquamous NSCLC patients in western countries and Asia [105,106], while a Phase II study in patients with KRAS mutations is currently enrolling [107].

Finally, results are awaited for a Phase I/II study with tivantinib, irinotecan and cetuximab in KRAS wild-type, pretreated metastatic colorectal cancer patients (TABLE 1) [47]. The Phase I trial portion determined tivantinib $360 \mathrm{mg}$ b.i.d. as the RP2D at full cetuximab dose, with ORR of $44 \%$, and among three MET-high patients, two partial responses and one stable disease have been observed. Interestingly, resistance to cetuximab has been associated to the activation of MET pathway in metastatic colorectal cancer [48].

\section{Conclusion}

Based on the above-mentioned studies, the anti-tumor activity of tivantinib is established in $\mathrm{HCC}$ as single agent and in combination with sorafenib. The main toxicities are asthenia, neutropenia, decreased appetite and anemia. The most pronounced clinical benefit was observed in MET-high HCC patients. As per our experience, the results of the Phase II trial in HCC represents the first randomized data reported with a MET inhibitor administered as a single agent in HCC, and the first identification of a biological subgroup of patients responding to a targeted therapy in HCC. Furthermore, MET expression was defined as a negative prognostic factor in second-line HCC. The Phase II results should be confirmed in a Phase III trial. 


\section{Future perspective \\ Predicting response to tivantinib: IHC \& FISH}

A crucial step in the development of molecularly targeted therapeutics lies in the identification of those patients most likely to benefit from treatment. Although some preclinical studies have shown that in cancer cells with activated HGF/MET autocrine loop or MET amplification, treatment with a MET TKI impairs the tumorigenic and metastatic potential [49]. Other data on MET inhibition appear somehow conflicting as they result from cell lines in which mechanisms of MET constitutive phosphorylation remain poorly understood [50]. Furthermore, the concept that sensitivity to MET inhibitors may have a genetic basis is challenged by the hypothesis of $M E T$ as an 'oncogene expedient', which amplifies the effect of other oncogenes even in the absence of specific genetic alterations. Assays to quantify HGF/MET expression and activity have not been extensively validated. Two different assays have been tested in order to identify which patients most benefit from tivantinib and other MET inhibitors: IHC and FISH. In addition to the aforementioned HCC and NSCLC Phase II trials [41,44], the Ventana anti-total MET immunohystochemical assay is currently adopted in an ongoing Phase III study of erlotinib in combination with an anti-MET moAb (onartuzumab) to select patients with MET diagnostic-positive NSCLC [108]. Recent data suggest that levels of MET expression might also predict a benefit from chemotherapy combined with an anti-HGF moAb (rilotumumab) in advanced gastroesophageal cancers [51]. Among 90 patients, $42 \%$ had a MET diagnostic-positive status: in this subgroup of patients, rilotumumab added to chemotherapy conferred clinically relevant increases of PFS and OS over placebo [51].

Cut-offs being adopted to define MET overexpression and antibodies used may vary across different series and cancer types, so that an unequivocal definition for MET overexpression is still lacking. While IHC provides relevant spatial and morphological information, tissue fixation and paraffin-embedding might hamper antibodies validation [52].

$M E T$ amplification on chromosome 7 may cause protein overexpression and a constitutive activation of the TK domain. MET copies as a predictor of response were retrospectively investigated by FISH in a Phase II randomized trial of tivantinib plus erlotinib versus placebo in advanced NSCLC [44]. Patients with four or more $M E T$ copies demonstrated a trend toward increased PFS under erlotinib plus tivantinib treatment. Benefit grew in magnitude as gene copy number increased, but statistical significance was not reached. Interestingly, MET:CEP7 ratios greater than two (which characteristically discriminate chromosome polysomy from true gene amplification) were observed in only three patients (2\%) [44]. Study outcomes were further explored according to MET protein expression by IHC [45]. However, in the intent-to-treat population, the benefit derived from erlotinib plus tivantinib over erlotinib plus placebo was observed in both MET-high and MET-low subgroups of patients [45]. Numerical abnormalities of chromosome 7 have been reported in HCC. MET amplification by FISH was reported in one of 44 HCC patients, whereas chromosome 7 polysomy was detected in an additional 17 patients [53]. It is unknown whether $M E T$ copy number might predict sensitivity to tivantinib in HCC. Nevertheless, these data seem to rule out the possibility that true $M E T$ amplification may play a role in predicting outcome to tivantinib in both NSCLC and HCC. By contrast, the correlation between chromosome 7 polysomy and outcome appears to be more sound and warrants further investigations.

\section{Other MET-targeted therapies under development in advanced HCC}

Currently, multiple anti-MET compounds are under study in HCC and in several tumor types, some of which are now entering Phase II and III trials. These targeted therapies include HGF and MET moAbs, which typically interfere with ligand and receptor interactions, and small-molecule TKIs directed to target receptor activation. Two additional classes of agents, in their early development stages, include decoys and antagonists that compete with ligand or receptor, and downstream inhibitors of MET.

Small-molecule TKIs can be further differentiated into selective TKIs, which specifically target MET in an ATP-competitive or noncompetitive manner, and unselective TKIs, which target MET as well as other kinases. Among selective MET TKIs being developed for advanced HCC, tivantinib has just completed the randomized Phase II trial stage. To our knowledge, no other selective MET TKI is being tested in similar development stages for second-line treatment of advanced HCC. Other highly selective MET TKIs, including EMD-1214063, LY-2801653, AMG-337, AMG 208 and INC-280, are rapidly 
entering Phase I studies. JNJ-38877605, a smallmolecule, ATP-competitive inhibitor of the catalytic activity of MET showed a 600-fold selectivity for MET in a panel of 250 tyrosine and serine-threonine kinases, and potently inhibited HGF-stimulated and constitutively activated MET phosphorylation [54]. A Phase I study with JNJ-38877605 was recently discontinued because of renal toxicities. Studies with the multi-target VEGFR and MET inhibitor XL184 (also known as cabozantinib) have shown significant activity against a number of solid tumors, including breast cancer, NSCLC, melanoma and castration-resistant prostate cancer. A Phase II discontinuation trial with XL184 in HCC patients has been recently presented [55]. In this study, where nearly half of patients were treatment-naive for advanced disease, the PFS results suggest a potential activity of this compound, independent from previous treatment. Interestingly, $38 \%$ of patients achieved a decline of AFP levels, which was demonstrated to correspond to an increase in survival in patients treated with sorafenib [56].

Other unselective MET TKIs include foretinib (XL880), which is an orally available compound that mainly inhibits MET and VEGFR2 [57]. The results of a recently published study show that molecular targeting of AXL, VEGFR2 and MET by foretinib efficiently inhibits the growth of HCC xenografts and significantly prolonged mouse survival [58]. A Phase I/II trial is ongoing in patients with advanced HCC [109].

\section{Financial \& competing interests disclosure}

The authors have no relevant affiliations or financial involvement with any organization or entity with a financial interest in or financial conflict with the subject matter or materials discussed in the manuscript. This includes employment, consultancies, honoraria, stock ownership or options, expert testimony, grants or patents received or pending, or royalties.

No writing assistance was utilized in the production of this manuscript.

\section{Executive summary}

\section{Mechanism of action}

- Tivantinib is a highly selective, orally administered, non-ATP-competitive mesenchymal-epithelial transition factor (MET) inhibitor, which strongly inhibits MET autoactivation by selectively targeting the inactive nonphosphorylated form of the kinase.

- Tivantinib arrests MET-dependent downstream signaling by disrupting constitutive and ligand-mediated MET phosphorylation.

\section{Pharmacokinetic properties}

- After oral administration, the pharmacokinetic profile of tivantinib is characterized by a moderate rate of absorption (peak 2-4 $\mathrm{h}$ after dosing), followed by a linear decrease in plasma concentrations approximately $4 \mathrm{~h}$ after dosing.

- A considerable interpatient variability in the pharmacokinetics of tivantinib was observed.

- Systemic tivantinib exposure generally grows with increasing doses, often less than proportionally.

- No relationships between drug-related adverse events, dose, baseline demographic variables and extent of tivantinib exposure were reported.

- Tivantinib is rapidly metabolized by CYP2C19 and moderately metabolized by CYP3A4, without inhibiting any of the major CYP450 enzymes.

\section{Clinical efficacy}

- In second-line hepatocellular carcinoma (HCC), tivantinib significantly increased time to progression compared with placebo.

- The most pronounced clinical benefits were observed in second-line MET-high HCC: tivantinib significantly increased time to progression and overall survival compared with placebo.

- In non-small-cell lung carcinomas prolonged progression-free survival compared with placebo was observed with tivantinib; the most pronounced benefit was observed in nonsquamous tumors.

\section{Safety \& tolerability}

- The main adverse events are asthenia, neutropenia, decreased appetite and anemia.

- In HCC, no significant increase of adverse events compared with placebo was observed, except for a higher incidence of hematologic events.

- In HCC, a manageable safety profile was observed with the dose of $240 \mathrm{mg}$ twice daily.

\section{Drug interactions}

- Coadministration of tivantinib with other anticancer agents does not alter the pharmacokinetic parameters of tivantinib, indicating an absence of drug-drug interactions.

\section{Dosage \& administration}

- In HCC, a regimen of tivantinib 240 mg twice daily was identified by the Phase II trial as the most appropriate.

- In other solid tumors, a regimen of tivantinib 360 mg twice daily, as monotherapy or in combination, was identified by Phase I trials as the most appropriate. 


\section{References}

Papers of special note have been highlighted as:

- of interest

- of considerable interest

1. Ferlay J, Shin HR, Bray F, Forman D, Mathers C, Parkin DM. Estimates of worldwide burden of cancer in 2008: GLOBOCAN 2008. Int. J. Cancer 127, 2893-2917 (2010).

2. Bruix J, Sherman M. Management of hepatocellular carcinoma: an update. Hepatology 53, 1020-1022 (2011).

3. European Association for the Study of the Liver, European Organisation for Research and Treatment of Cancer. EASL-EORTC Clinical Practice Guidelines: management of hepatocellular carcinoma. J. Hepatol. 56, 908-943 (2012).

4. Forner A, Llovet JM, Bruix J. Hepatocellular carcinoma. Lancet 379, 1245-1255 (2012).

5. Llovet JM, Ricci S, Mazzaferro V et al. Sorafenib in advanced hepatocellular carcinoma. N. Engl. J. Med. 359, 378-390 (2008).

6. Cheng AL, Kang YK, Chen Z et al. Efficacy and safety of sorafenib in patients in the Asia-Pacific region with advanced hepatocellular carcinoma: a Phase III randomised, double-blind, placebo-controlled trial. Lancet Oncol. 10, 25-34 (2009).

7. Comoglio PM, Giordano S, Trusolino L. Drug development of MET inhibitors: targeting oncogene addiction and expedience. Nat. Rev. Drug Discov. 7, 504-516 (2008).

8. Eder JP, Vande Woude GF, Boerner SA, LoRusso PM. Novel therapeutic inhibitors of the c-Met signaling pathway in cancer. Clin. Cancer Res. 15, 2207-2214 (2009).

9. Boccaccio C, Comoglio PM. Invasive growth: a MET-driven genetic programme for cancer and stem cells. Nat. Rev. Cancer 6, 637-645 (2006).

10. Suzuki K, Hayashi N, Yamada Y et al. Expression of the c-Met protooncogene in human hepatocellular carcinoma. Hepatology 20, 1231-1236 (1994).

11. Ueki T, Fujimoto J, Suzuki T, Yamamoto H, Okamoto E. Expression of hepatocyte growth factor and its receptor c-met proto-oncogene in hepatocellular carcinoma. Hepatology 25, 862-866 (1997).

12. Osada S, Kanematsu M, Imai H, Goshima S. Clinical significance of serum HGF and c-Met expression in tumor tissue for evaluation of properties and treatment of hepatocellular carcinoma. Hepatogastroenterology 55, 544-549 (2008).

13. Kaposi-Novak P, Lee JS, Gòmez-Quiroz L, Coulouarn C, Factor VM, Thorgeirsson SS. Met-regulated expression signature defines a subset of human hepatocellular carcinomas with poor prognosis and aggressive phenotype. J. Clin. Invest. 116(6), 1582-1595 (2006).

14. Zhang SZ, Pan FY, Xu JF et al. Knockdown of c-Met by adenovirus-delivered small interfering RNA inhibits hepatocellular carcinoma growth in vitro and in vivo. Mol. Cancer Ther. 4, 1577-1584 (2005).

15. Salvi A, Arici B, Portolani N, Giulini SM, De Petro G, Barlati S. In vitro c-met inhibition by antisense RNA and plasmidbased RNAi down-modulates migration and invasion of hepatocellular carcinoma cells. Int. J. Oncol. 31, 451-460 (2007).

16. Faivre S, Raymond E, Boucher E et al. Safety and efficacy of sunitinib in patients with advanced hepatocellular carcinoma: an openlabel, multicentre, Phase II study. Lancet Oncol. 10(8), 794-800 (2009).

17. Zhu AX, Sahani DV, Duda DG et al. Efficacy, safety, and potential biomarkers of sunitinib monotherapy in advanced hepatocellular carcinoma: a Phase II study. J. Clin. Oncol. 27(18), 3027-3035 (2009).

18. Cheng A, Kang Y, Lin D et al. Phase III trial of sunitinib $(\mathrm{Su})$ versus sorafenib (So) in advanced hepatocellular carcinoma (HCC). Presented at: 2011 American Society Clinical Onology Annual Meeting. Chicago, IL, USA, 3-7 June 2011 (Abstract 4000).

19. Llovet JM, Decaens T, Raoul J-L et al. Brivanib vs placebo in patients with advanced hepatocellular carcinoma (HCC) who failed or were intolerant to sorafenib: results from the Phase 3 BRISK-PS study. Presented at: 47th European Association for the Study of the Liver International Liver Congress. Barcelona, Spain, 18-22 April 2012 (Abstract 1398).

20. Villanueva A, Toffanin S, Llovet JM. Linking molecular classification of hepatocellular carcinoma and personalized medicine: preliminary steps. Curr. Opin. Oncol. 20(4), 444-453 (2008).

21. Abou-Alfa GK, Schwartz L, Ricci S et al. Phase II study of sorafenib in patients with advanced hepatocellular carcinoma. J. Clin. Oncol. 24(26), 4293-3000 (2006).

22. Zhang Z, Zhou X, Shen H, Wang D, Wang Y. Phosphorylated ERK is a potential predictor of sensitivity to sorafenib when treating hepatocellular carcinoma: evidence from an in vitro study. BMC Med. 7, 41 (2009).

23. Llovet JM, Peña CE, Lathia CD, Shan M, Meinhardt G, Bruix J; SHARP Investigators Study Group. Plasma biomarkers as predictors of outcome in patients with advanced hepatocellular carcinoma. Clin. Cancer Res. 18(8), 2290-2300 (2012).
24. Personeni N, Rimassa L, Santoro A. Biomarkers in hepatocellular carcinoma - letter. Clin. Cancer Res. 18(17), 4861 (2012).

25. Munshi N, Jeay S, Li Y et al. ARQ197, a novel and selective inhibitor of the human c-Met receptor tyrosin kinase with antitumor activity. Mol. Cancer Ther. 9 , 1544-1553 (2010).

- First report describing the discovery of tivantinib as the first non-ATP-competitive small molecule that selectively targets mesenchymal-epithelial transition factor (MET) receptor tyrosine kinase. This study explores the activity of tivantinib against a wide range of human cancer cell lines, its pharmacodynamic properties in vitro and in vivo, and its effects in multiple mouse xenograft models.

26. Chen CR, Szwaya J, Rojnukarin A et al. Combination studies of tyrosine kinase inhibitors (TKIs): assessment of potential cytotoxic synergy of ARQ 197 with sorafenib or sunitinib. Presented at: 100th American Association for Cancer Research Annual Meeting 2009. Denver, CO, USA, 18-22 April 2009 (Abstract 820).

27. Pant S, Saleh M, Bendell J et al. Phase 1B dose escalation trial evaluating c-MET inhibitor ARQ 197 plus gemcitabine in patients with advanced solid tumors on schedules refined by in vitro mechanistic studies. Presented at: 35th European Society of Medical Oncology Annual Meeting. Milan, Italy, 8-12 October 2010 (Abstract 510P).

28. Previdi S, Abbadessa G, Dalò F, France DS, Broggini M. Breast cancer-derived bone metastasis can be effectively reduced through specific c-MET inhibitor tivantinib (ARQ 197) and shRNA c-MET knockdown. Mol. Cancer Ther. 11(1), 214-223 (2012).

29. Savage RE, Zhong $\mathrm{C}$, Hall $\mathrm{T}$ et al. In vitro ADME Properties of ARQ 197. Comparison to in vivo data. Presented at: 99th American Association for Cancer Research Annual Meeting. Philadelphia, PA, USA, 11-15 April 2008 (Poster 1291).

30. Rosen LS, Senzer N, Mekhail T et al. A Phase I dose-escalation study of Tivantinib (ARQ 197) in adult patients with metastatic solid tumors. Clin. Cancer Res. 17(24), 7754-7764 (2011).

31. Yap TA, Olmos D, Brunetto AT et al. Phase I trial of a selective c-MET inhibitor ARQ-197 incorporating proof of mechanism pharmacodynamic studies. J. Clin. Oncol. 29, 1271-1279 (2011).

- Defines the maximum tolerated dose and recommended Phase II dose of tivantinib at $360 \mathrm{mg}$ twice daily. 
32. Santoro A, Simonelli M, Rodriguez-Lope C et al. A Phase-1b study of tivantinib (ARQ 197) in adult patients with hepatocellular carcinoma and cirrhosis. Br. J. Cancer 108(1), 21-24 (2013).

33. Goldman JW, Laux I, Chai F et al. Phase 1 dose-escalation trial evaluating the combination of the selective MET (mesenchymal-epithelial transition factor) inhibitor tivantinib (ARQ 197) plus erlotinib. Cancer 118(23), 5903-5911 (2012).

34. Adjei AA, Sosman JA, Dy GK et al. A Phase I dose-escalation trial evaluating ARQ 197 administered in combination with sorafenib in adult patients (pts) with advanced solid tumors. Presented at: 46th American Society of Clinical Oncology Annual Meeting. Chicago, IL, USA, 4-8 June 2010 (Abstract 3024).

35. Engelman JA, Zejnullahu K, Mitsudomi T et al. MET amplification leads to gefitinib resistance in lung cancer by activating ERBB3 signaling. Science 316, 1039-1043 (2007).

36. Martel R, Puzanov I, When WM et al. Safety and efficacy of MET inhibitor tivantinib (ARQ 197) combined with sorafenib in patients (pts) with hepatocellular carcinoma (HCC) from a Phase I study. Presented at: 48th American Society of Clinical Oncology Annual Meeting. Chicago, IL, USA, 1-5 June 2012 (Abstract 4117).

37. Means-Powell JA, Adjei AA, Puzanov I et al. Safety and efficacy of MET inhibitor tivantinib (ARQ 197) combined with sorafenib in patients (pts) with NRAS wild-type or mutant melanoma from a Phase I study. Presented at: 48th American Society of Clinical Oncology Annual Meeting. Chicago, IL, USA, 1-5 June 2012 (Abstract 8519).

38. Puzanov I, Sosman JA, Santoro A et al. Safety and efficacy of MET inhibitor tivantinib (ARQ 197) combined with sorafenib in patients (pts) with renal cell carcinoma (RCC) from a Phase I study. Presented at: 48th American Society of Clinical Oncology Annual Meeting. Chicago, IL, USA, 1-5 June 2012 (Abstract 4545).

39. Camacho LH, Pant S, Saleh MN et al. Phase Ib results of c-MET inhibitor ARQ 197 in combination with gemcitabine in a cohort of patients (pts) with advanced breast, ovarian, and uterine tumors. Presented at: 47th American Society of Clinical Oncology Annual Meeting. Chicago, IL, USA, 3-7 June 2011 (Abstract 3077).

40. Camacho LH, Bendell JC, Pant S et al. Phase $1 \mathrm{~b}$ Results of c-MET inhibitor Tivantinib (ARQ 197) in combination with Gemcitabine in a cohort of patients with advanced thoracic tumors. Presented at: 14 th World Conference on Lung Cancer. Amsterdam RAI, The Netherlands, 3-7 July 2011 (Abstract).

41. Santoro A, Rimassa L, Borbath I et al. Tivantinib for second-line treatment of advanced hepatocellular carcinoma: a randomised, placebo-controlled Phase 2 study. Lancet Oncol. 14(1), 55-63 (2013).

- First report of the randomized Phase II study of tivaninib in second-line hepatocellular carcinoma. The primary end point was met, giving the most pronounced benefit shown in MET-high patients. First identification of a biological subgroup of patients responding to a targeted therapy in hepatocellular carcinoma.

42. Eisenhauer EA, Therasse P, Bogaerts J et al. New response evaluation criteria in solid tumours: revised RECIST guideline (version 1.1). Eur. J. Cancer 45(2), 228-247 (2009).

43. Cappuzzo F, Marchetti A, Skokan M et al. Increased MET gene copy number negatively affects survival of surgically resected non-small-cell lung cancer patients. J. Clin. Oncol. 27, 1667-1674 (2009).

44. Sequist LV, von Pawel J, Garmey EG et al. Randomized Phase II study of erlotinib plus tivantinib versus erlotinib plus placebo in previously treated non-small-cell lung cancer. J. Clin. Oncol. 29(24), 3307-3315 (2011).

- Randomized Phase II study of erlotinib plus or minus tivantinib in pretreated non-small-cell lung carcinoma.

45. Rodig S, Sequist LV, Schiller JH et al. An exploratory biomarker analysis evaluating the effect of the c-MET inhibitor tivantinib (ARQ 197) and erlotinib in NSCLC patients in a randomized, double-blinded Phase 2 study. Presented at: 103rd American Association for Cancer Research Annual Meeting. Chicago, IL, USA, 31 March-4 April 2012 (Abstract 1729).

- Retrospective evaluation of MET by immunohistochemistry and FISH to predict the efficacy of erlotinib with or without tivantinib in non-small-cell lung carcinoma.

46. Ma PC, Jagadeeswaran R, Jagadeesh S et al. Functional expression and mutations of c-Met and its therapeutic inhibition with SU11274 and small interfering RNA in nonsmall cell lung cancer. Cancer Res. 65(4), 1479-1488 (2005).

47. Eng C, Bessudo A, Gabrail N et al. Phase I/II study of tivantinib (ARQ 197), irinotecan and cetuximab in patients with KRAS wild-type, previously treated, metastatic colorectal cancer. Presented at: 14th World Congress on Gastrointestinal Cancer. Barcelona, Spain, 27-30 June 2012 (Abstract PD-0018).

48. Inno A, Di Salvatore M, Cenci T et al. Is there a role for IGF1R and c-MET pathways in resistance to cetuximab in metastatic colorectal cancer? Clin. Colorectal Cancer 10(4), 325-332 (2011).

49. Pan BS, Chan GK, Chenard M et al. MK-2461, a novel multitargeted kinase inhibitor, preferentially inhibits the activated c-Met receptor. Cancer Res. 70 (4), 1524-1533 (2010).

50. You H, Ding W, Dang H, Jiang Y, Rountree CB. C-Met represents a potential therapeutic target for personalized treatment in hepatocellular carcinoma. Hepatology 54(3), 879-889 (2011)

- Preclinical evidence and implications for MET inhibition in hepatocellular carcinoma.

51. Oliner KS, Tang R, Anderson A et al. Evaluation of MET pathway biomarkers in a Phase II study of rilotumumab ( $\mathrm{R}, \mathrm{AMG}$ 102) or placebo (P) in combination with epirubicin, cisplatin, and capecitabine (ECX) in patients (pts) with locally advanced or metastatic gastric (G) or esophagogastric junction (EGJ) cancer. Presented at: 2012 American Society of Clinical Oncolocy Annual Meeting. Chicago, IL, USA, 1-5 June 2012 (Abstract 4005).

52. Pozner-Moulis S, Cregger M, Camp RL, Rimm DL. Antibody validation by quantitative analysis of protein expression using expression of Met in breast cancer as a model. Lab. Invest. 87(3), 251-260 (2007).

53. Kondo $\mathrm{S}$, Ojima H, Tsuda $\mathrm{H}$ et al. Clinical impact of c-Met expression and its gene amplification in hepatocellular carcinoma. Int. J. Clin. Oncol. doi: 10.1007/s10147-0110361-95 (2012) (Epub ahead of print).

54. Perera T, Lavrijssen T, Janssens B et al. JNJ-38877605: a selective Met kinase inhibitor inducing regression of Met-driven tumor models. Presented at: 99th American Association for Cancer Research Annual Meeting. Philadelphia, PA, USA, 11-15 April 2008 (Abstract 4837).

55. Verslype C, Cohn AL, Kelley RK et al. Activity of cabozantinib (XL184) in hepatocellular carcinoma: results from a Phase II randomized discontinuation trial (RDT). Presented at: 2012 Gastrointestinal Cancers Symposium. San Francisco, CA, USA, 19-21 January 2012 (Abstract 4007).

56. Personeni N, Bozzarelli S, Pressiani T et al. Usefulness of alpha-fetoprotein response in patients treated with sorafenib for advanced hepatocellular carcinoma. J. Hepatol. 57(1), 101-107 (2012). 
57. Qian F, Engst $S$, Yamaguchi K et al. Inhibition of tumor cell growth, invasion, and metastasis by EXEL-2880 (XL880, GSK1363089), a novel inhibitor of HGF and VEGF receptor tyrosine kinases. Cancer Res. 69(20), 8009-8016 (2009).

58. Huynh H, Ong R, Soo KC. Foretinib demonstrates anti-tumor activity and improves overall survival in preclinical models of hepatocellular carcinoma. Angiogenesis 15(1), 59-70 (2012).

\section{Websites}

101. ClinicalTrials.gov. A study of ramucirumab (IMC-1121B) drug product (DP) and best supportive care (BSC) versus placebo and BSC as 2nd-line treatment in patients with hepatocellular carcinoma after 1st-line therapy with sorafenib (2012). http://clinicaltrials.gov/ct2/show/NCT0114 0347? term $=$ NCT01140347\&rank=1
102. ClinicalTrials.gov. Global study looking at the combination of RAD001 plus best supportive care (BSC) and placebo plus BSC to treat patients with advanced hepatocellular carcinoma (2012).

http://clinicaltrials.gov/ct2/show/NCT0103 5229? term $=$ NCT01035229 \& rank=1

103. ArQule, Inc. www.arqule.com

104. Daiichi Sankyo, Inc. www.daiichisankyo.com

105. ClinicalTrials.gov. ARQ 197 plus erlotinib versus placebo plus erlotinib for the treatment of non-squamous, non-small-cell lung cancer (2012).

http://clinicaltrials.gov/ct2/show/NCT0124 4191? term $=$ NCT01244191\&rank=1

106. ClinicalTrials.gov. A Phase 3, randomized, double-blinded, placebo-controlled study of ARQ 197 plus erlotinib versus placebo plus erlotinib (2012).
http://clinicaltrials.gov/ct2/show/NCT0137 7376? term $=$ NCT01377376\&rank $=1$

107. ClinicalTrials.gov. Erlotinib plus ARQ 197 versus single agent chemotherapy in locally advanced or metastatic non-small cell lung cancer (2012).

http://clinicaltrials.gov/ct2/show/NCT0139 5758 ? term $=$ NCT01395758 \& rank $=1$

108. ClinicalTrials.gov. A study of onartuzumab (MetMAb) in combination with tarceva (erlotinib) in patients with met diagnosticpositive non-small cell lung cancer who have received chemotherapy for advanced or metastatic disease (MetLung) (2012). http://clinicaltrials.gov/ct2/show/NCT0145 6325?term = NCT01456325\&rank=1

109. ClinicalTrials.gov. Safety study of foretinib (GSK1363089) in adults with liver cancer (2012).

http://clinicaltrials.gov/ct2/show/NCT0092 0192? term $=$ NCT00920192 \& $\mathrm{rank}=1$ 